YITP-SB-99-68

hep-th/0001051

Nov, 1999

\title{
Hyperkähler Quotients, Mirror Symmetry, and F-theory
}

\author{
I.Y. Park* and R. von Unge** \\ * C.N. Yang Institute for Theoretical Physics \\ State University of New York \\ Stony Brook, N. Y. 11794-3840 \\ ** Department of Theoretical Physics and Astrophysics \\ Faculty of Science, Masaryk University \\ Kotlářská 2, CZ-611 37, Brno, Czech Republic
}

\begin{abstract}
Using the graphical method developed in hep-th/9908082, we obtain the full curve corresponding to the hyperkähler quotient from the extended $E_{7}$ Dynkin diagram. As in the $E_{6}$ case discussed in the same paper above, the resulting curve is the same as the one obtained by Minahan and Nemeschansky. Our results seem to indicate that it is possible to define a generalized Coulomb branch such that four dimensional mirror symmetry would act by interchanging the generalized Coulomb branch with the Higgs branch of the dual theory. To understand these phenomena, we discuss mirror symmetry and F-theory compactifications probed by D3 branes.
\end{abstract}

${ }^{1}$ email: ipark@insti.physics.sunysb.edu

${ }^{2}$ email:unge@physics.muni.cz 


\section{Introduction}

String theory provides a very powerful setting for the study of gauge theories. Gauge theories can be constructed through the geometrical engineering [1] by compactifying string theory on Calabi-Yau manifolds with appropriate Hodge numbers and singularities. They can also be realized as the world volume theories on extended objects such as D-branes [2]. The authors of [3] studied the gauge theories obtained by placing D-branes on orbifold singularities and introduced 'quiver diagrams', which summarize the gauge group structures and matter contents of the gauge theories. They considered D-5 branes and noticed that the moduli space of D-brane ground states is a ALE space described by a hyperkähler quotient.

The hyperkähler quotient was introduced in [4]; it was mathematically refined in [5]. One way to construct it is to gauge isometries of a non-linear sigma model in such a way as to preserve $\mathcal{N}=2$ supersymmetry. In the spirit of [6], a graphical method was invented in [7] and used to obtain the curve that corresponds to a hyperkähler quotient of a linear space. In particular, it was applied to the hyperkähler quotients constructed from the extended Dynkin diagrams of $\mathrm{A}_{k}, \mathrm{D}_{k}$ series and $\mathrm{E}_{6}$ case.

Remarkably, the Higgs branch of a quiver gauge theory based on the extended $\mathrm{E}_{6}$ Dynkin diagram turned out to be identical, when it was expressed in terms of $\mathrm{E}_{6}$ Casimir invariants, to the curve円 with $\mathrm{E}_{6}$ global symmetry obtained by Minahan and Nemeschansky some time ago [8] and later by the authors of [10]. In this article, we work out the full[ curve corresponding to the $\mathrm{E}_{7}$ extended Dynkin diagram. The resulting curve is again equal to the generalized Coulomb branch with $\mathrm{E}_{7}$ global symmetry computed in [9] and [10].

We understand the origin of these phenomena through mirror symmetry and F-theory [11] compactifications [12, 13]. D3 branes are used to probe the singularities of the backgrounds under consideration [14]. The relevant F-theory compactifications for our purpose are the ones which give rise to $\mathrm{E}_{7}$ gauge group. The $\mathrm{E}_{7}$ global symmetry is realized on the world volume theory of the D3 branes.

It is the physics near such singularities that is responsible for the field theory limit of string compactifications [15]. The mirror geometry of ADE singularities was discussed [16] in the context of type II strings. On the dual backgrounds, the gauge groups of the dual superconformal field theories are given by a product of $\mathrm{U}\left(\mathrm{n}_{i}\right)$ groups. The $\mathrm{n}_{i}$ 's are given by multiples of the Dynkin numbers of the nodes in the corresponding Dynkin diagrams.

\footnotetext{
${ }^{1}$ We will call this curve the generalized Coulomb branch.

${ }^{2}$ The orbifold limits of $\mathrm{E}_{7}$ and $\mathrm{E}_{8}$ (and some other higher order quiver diagrams) were considered in $[7$.

${ }^{3} \mathrm{In}_{7}$ case, it is easier to compare with [10] since the authors used $\mathrm{E}_{7}$ Casimir invariants, while the authors of [9] expressed their curve in terms of the $\mathrm{SO}(12) \times \mathrm{SU}(2)$ Casimir invariants, as we discussed in section 2 .
} 
Mirror symmetry is well understood in three dimensions [17, 18] where both the Higgs branch and the Coulomb branch are hyperkähler manifolds. They get interchanged under the action of mirror symmetry. In the four dimensional models we consider in this paper, the Coulomb branch of the original theory is a Riemann surface, which is real two dimensional, whereas the Higgs branch of the dual theory has real four dimensions. What we find in this paper seems to indicate that mirror symmetry in these four dimensional models acts in such a way that it is the generalized Coulomb branch (rather than the Coulomb branch) of the original gauge theory that gets interchanged with the Higgs branch of the mirror dual theory.

More intuitive understanding of the origin of the identity between the curve we compute and the generalized Coulomb branch seems possible by applying various string dualities to the system under consideration. We briefly discuss this point in section 3 with a heuristic example using the D7-D3 brane system.

The organization is as follows. After briefly reviewing the hyperkähler quotients, we present the calculation of $\mathrm{E}_{7}$ case in section 2. The final form of the curve is given in Appendix A. It is expressed in terms of $\mathrm{E}_{7}$ Casimirs, $P_{i}$, whose definition is given in Appendix A. As in the case of $\mathrm{E}_{6}$, the curve obtained is the generalized Coulomb branch with $\mathrm{E}_{7}$ global symmetry. The generalized Coulomb branch can also be expressed in terms of $\mathrm{E}_{7}$ Casimir invariants which we also denote as $P_{i}$. However, the $P_{i}$ 's of our curve are functions of Fayet-Iliopoulos (FI) parameters, $\mathrm{b}_{j}$, while the $P_{i}$ 's of the generalized Coulomb branch are functions of mass parameters, $\mathrm{m}_{k}$. In anticipation of mirror symmetry, we find the relations between b's and m's which render $P_{i}(b)=P_{i}(m)$. [. In section 3, we discuss mirror symmetry and F-theory compactifications probed by D3 branes. Section 4 includes summary and open problems.

\section{The Hyperkähler Quotient For $\mathrm{E}_{7}$ Case}

We begin by briefly reviewing the hyperkähler quotients and refer the reader to [4, 5, 4] (and the references therein) for more details. Intuitively speaking the (hyper)kähler quotient is a method that, starting with a space with a metric that has some isometry, finds a hypersurface orthogonal to the isometry direction and the induced metric on that surface. The method used in this paper will allow us to explicitly find this hypersurface as a two dimensional

\footnotetext{
${ }^{4}$ There is a natural relation between the generalized Coulomb branch in four dimensions and the Coulomb branch in three dimensions, as discussed in section 3.

${ }^{5}$ They are associated with relevant deformations of the superconformal field theory under consideration.

${ }^{6}$ These relations reflect the fact that under mirror symmetry FI and mass parameters get interchanged.
} 
complex space embedded in $C^{3}$. It will also allow us to find the explicit dependence of this space on the Fayet-Iliopoulos parameters of the gauge theory we start with. In general, turning them on we deform the hypersurface so that it becomes non-singular. To also find the induced metric in the hyperkähler quotient one should introduce a non-linear sigma model with the original space as its target space. Gauging the isometries of this non-linear sigma model while preserving $\mathcal{N}=2$ susy gives rise to the hyperkähler quotient. More specifically, consider a sigma model with isometries. To elevate the isometries to local symmetries, introduce an $\mathcal{N}=2$ vector multiplet, which consists of an $\mathcal{N}=1$ vector multiplet and an $\mathcal{N}=1$ chiral multiplet, denoted respectively as $V, S$ in $[1$. In $\mathcal{N}=1$ superspace, one integrates out $V$ and $S$ by their field equations. Inserting the solution for $V$ field equations into the gauged Lagrangian and keeping the $S$ field equations as constraints gives the Kähler potential of the quotient space. The constraints from $S$ field equations can be represented graphically and are given in figure $1^{7}$. The gauge groups and the representations appropriate for the the construction of ALE spaces are summarized by the extended Dynkin diagrams [19], as in Figure 2.

Now, we compute the hyperkähler quotient corresponding to the $\mathrm{E}_{7}$ extended Dynkin Diagram given in Figure 3(a). In addition to the Dynkin numbers in the same figure, we label the nodes by assigning 1 to the far left node and 2 to the next one, etc. The upper node in the middle is referred to as the eighth node. We closely follow [7] with a convenient set of variables defined in Figure 3(b).

Consider the highest order invariant, $U$, and its orientation reversed diagram $\bar{U}$. The product of these two diagrams can be written as

$$
U \bar{U}=W \operatorname{Tr}(M N K N)
$$

One can use the so called Schouten identity to rewrite $\bar{U}$ in terms of the variables defined in Figure 3(b): The relevant Schouten identity is

$$
\operatorname{Tr}(\{M, N\} K)=\operatorname{Tr}(M N) \operatorname{Tr}(K)+\operatorname{Tr}(M K) \operatorname{Tr}(N)+\operatorname{Tr}(N K) \operatorname{Tr}(M)-\operatorname{Tr}(M) \operatorname{Tr}(N) \operatorname{Tr}(K)
$$

Noting the following relations,

$$
\begin{aligned}
\operatorname{Tr}(\{M, N\} K) & =U+\bar{U} \\
\operatorname{Tr}(M) & =b_{1}
\end{aligned}
$$

\footnotetext{
7 Figure 1 and Figure 3 are taken from $[7]$.

${ }^{8}$ In the orbifold limit, $U=-\bar{U}$, but this is not true in the presence of the Fayet-Iliopoulos terms.
} 


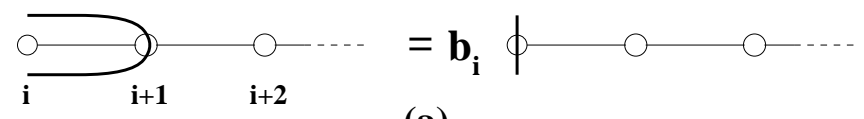

(a)

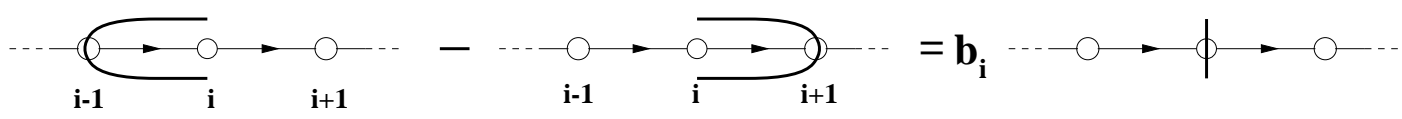

(b)

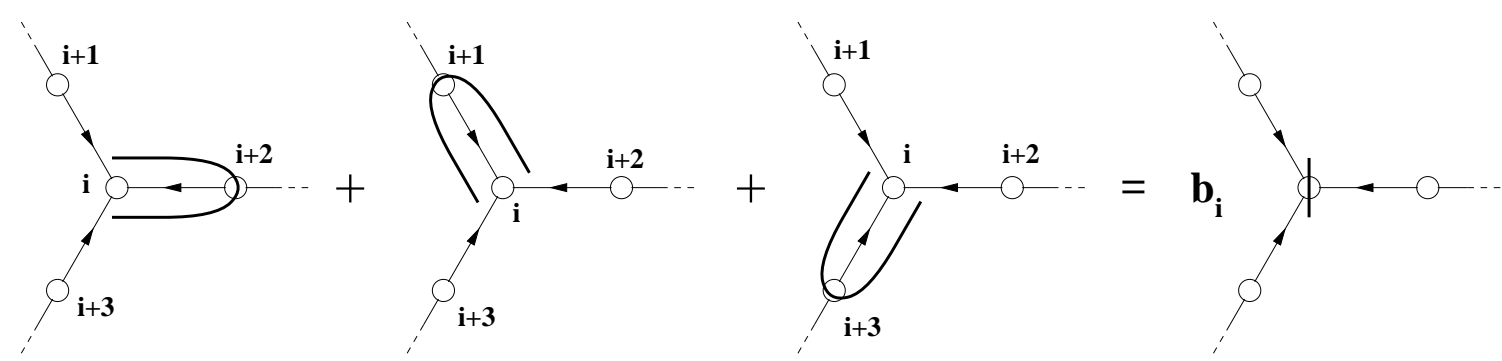

(c)

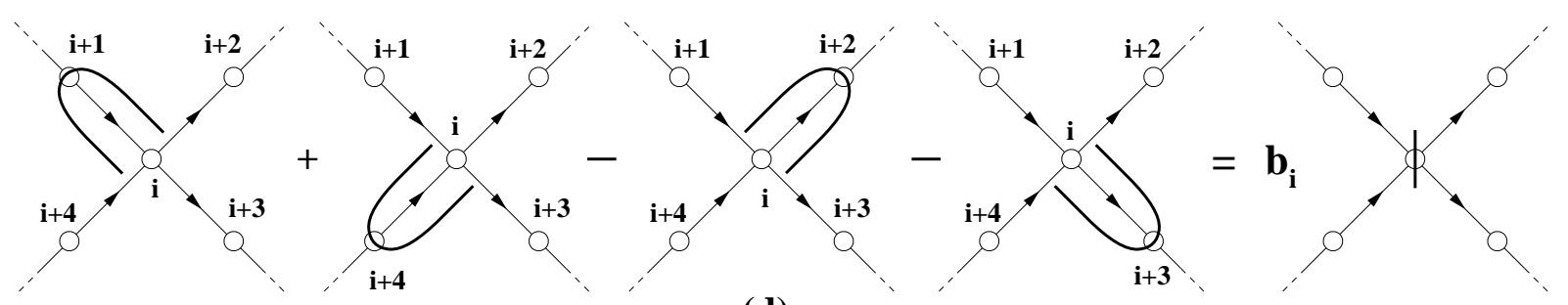

(d)

Figure 1: The bug calculus. $b_{i}$ is the Fayet-Iliopoulos parameter associated to the $i$ 'th node, and a vertical bar through the $i$ 'th node represents a $U\left(N_{i}\right)$ Kronecker- $\delta$.
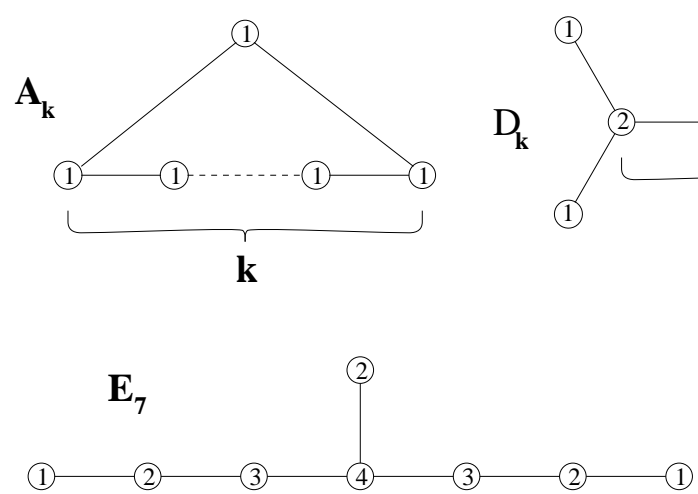
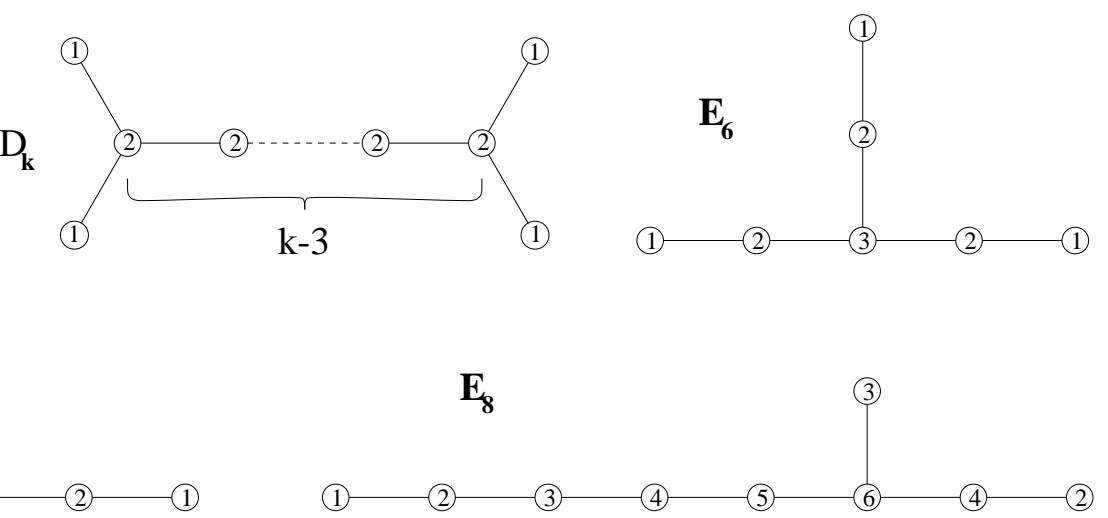

Figure 2 


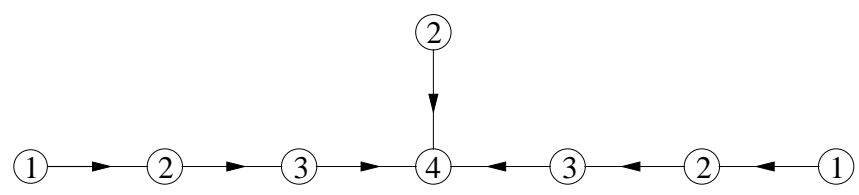

(a)
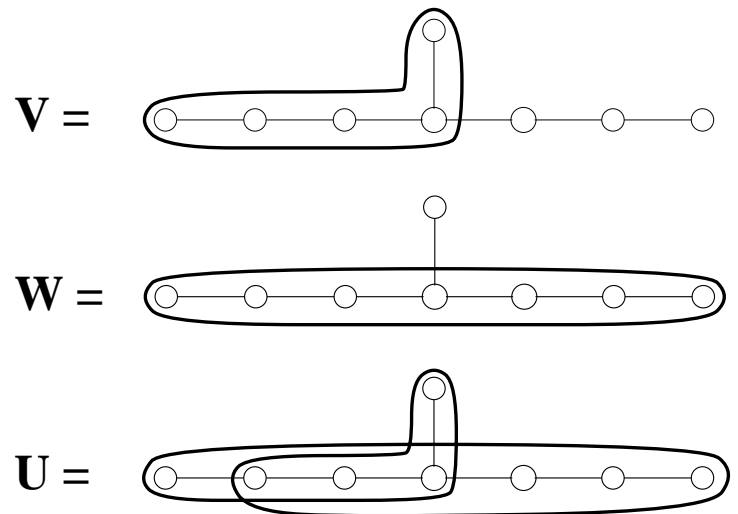

(b)
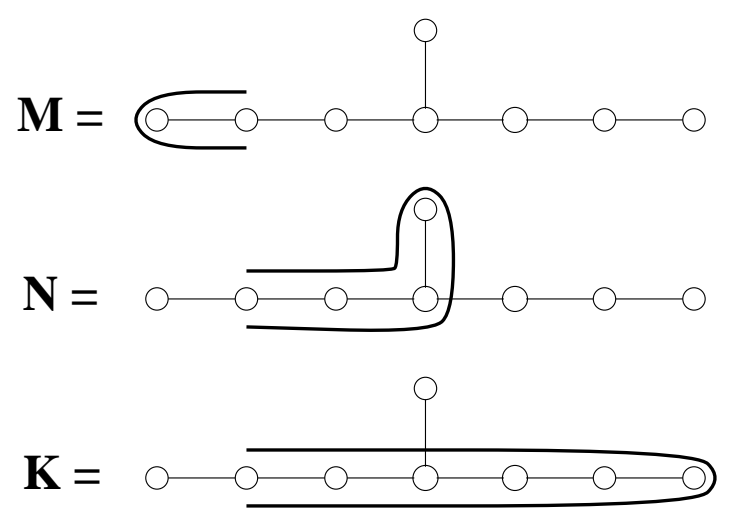

(c)

Figure 3: The $E_{7}$ invariants and some useful matrices.

$$
\begin{aligned}
& \operatorname{Tr}(M N)=V \\
& \operatorname{Tr}(M K)=W
\end{aligned}
$$

one obtains,

$$
\bar{U}=-U+\operatorname{Tr}(K) V+\operatorname{Tr}(N) W+b_{1} \operatorname{Tr}(N K)-b_{1} \operatorname{Tr}(N) \operatorname{Tr}(K)
$$

where $b_{1}$ is the FI term associated with the first node of the Dynkin diagram. To rewrite the right hand side of (11), consider

$$
\begin{aligned}
0= & N_{k_{1}}^{\left[k_{2}\right.} K_{k_{2}}^{k_{3}} N_{k_{3}}^{\left.k_{4}\right]} M_{k_{4}}^{k_{1}} \\
= & \operatorname{Tr}(M N K N)+\operatorname{Tr}(N N K M)-\operatorname{Tr}(N N M) \operatorname{Tr}(K)-\operatorname{Tr}(N K M) \operatorname{Tr}(N) \\
& +\operatorname{Tr}(N M) \operatorname{Tr}(K) \operatorname{Tr}(N)-\operatorname{Tr}(N M) \operatorname{Tr}(K N)
\end{aligned}
$$

Applying the Schouten identity (22) again to $\operatorname{Tr}(N N K N)$ and $\operatorname{Tr}(N N M)$ leads to

$$
\operatorname{Tr}(M N K N)=\operatorname{Tr}(M N) \operatorname{Tr}(N K)-\frac{1}{2} \operatorname{Tr}(M K) \operatorname{Tr}(N N)+\frac{1}{2} \operatorname{Tr}(M K) \operatorname{Tr}(N)^{2}
$$




$$
\begin{aligned}
+\frac{1}{2} \operatorname{Tr}(N N) \operatorname{Tr}(M) \operatorname{Tr}(K)-\frac{1}{2} \operatorname{Tr}(M) \operatorname{Tr}(K) \operatorname{Tr}(N)^{2} \\
=\operatorname{Tr}(N K) V+\left[-\frac{1}{2} \operatorname{Tr}(N N)+\operatorname{Tr}(N)^{2}\right] W \\
+\frac{b_{1}}{2} \operatorname{Tr}(K) \operatorname{Tr}(N N)-\frac{b_{1}}{2} \operatorname{Tr}(K) \operatorname{Tr}(N)^{2}
\end{aligned}
$$

where the second equality follows from (3). Substituting (4) and (6) into (目) gives

$$
\begin{aligned}
& U\left(-U+\operatorname{Tr}(K) V+\operatorname{Tr}(N) W+b_{1} \operatorname{Tr}(N K)-b_{1} \operatorname{Tr}(N) \operatorname{Tr}(K)\right) \\
& =W\left(\operatorname{Tr}(N K) V+\left[-\frac{1}{2} \operatorname{Tr}(N N)+\operatorname{Tr}(N)^{2}\right] W+\frac{b_{1}}{2} \operatorname{Tr}(K) \operatorname{Tr}(N N)-\frac{b_{1}}{2} \operatorname{Tr}(K) \operatorname{Tr}(N)^{2}\right)
\end{aligned}
$$

Therefore the whole task of finding the curve is reduced to the computation of $\operatorname{Tr}(N), \operatorname{Tr}(K)$, $\operatorname{Tr}(N N)$ and $\operatorname{Tr}(N K)$. It is a simple exercise to compute $T(N)$ : It is expressed purely in terms of $b_{i}$ 's. The other three quantities are more complicated to obtain: The final forms are,

$$
\begin{aligned}
\operatorname{Tr}(K) & =k_{v}\left(b_{i}\right) V+k \\
\operatorname{Tr}(N N) & =2 W+n_{v}\left(b_{i}\right) V+n\left(b_{i}\right) \\
\operatorname{Tr}(N K) & =m_{w}\left(b_{i}\right) W-V^{2}+m_{v}\left(b_{i}\right) V+m\left(b_{i}\right)
\end{aligned}
$$

where the coefficients are functions of the FI parameters, $b_{i}$, as indicated. Since these coefficients are lengthy, we will not present them explicitly. However, once we make the change of variables discussed below, the coefficients can be expressed in terms of $\mathrm{E}_{7}$ Casimir invariants. This makes the curve simple enough to present. Upon substitution of (8) into (घ), we obtain,

$$
\begin{aligned}
& U^{2}-W^{3}-V^{3} W \\
& +U\left(\left(-b_{1} m_{w}-l\right) W+\left(b_{1}-k_{v}\right) V^{2}+\left(-b_{1} m_{v}+b_{1} l k_{v}-k\right) V+b_{1}(l k-m)\right) \\
& +W^{2}\left(\left[m_{w}-\frac{1}{2} n_{v}+b_{1} k_{v}\right] V+\frac{1}{2} l^{2}+b_{1} k-\frac{n}{2}\right) \\
& +W\left(\left[\frac{b_{1}}{2} n_{v} k_{v}+m_{v}\right] V^{2}+\left[m+\frac{b_{1}}{2} n k_{v}+\frac{b_{1}}{2} n_{v} k-\frac{b_{1}}{2} l^{2} k_{v}\right] V-\frac{b_{1}}{2} l^{2} k+\frac{b_{1}}{2} n k\right)=0
\end{aligned}
$$

where $l \equiv \operatorname{Tr}(N)$. To put this curve into the standard form, we perform the following change of variables, 


$$
\begin{array}{r}
U=\quad X-\frac{1}{2}\left[\left(b_{1} m_{w}-l\right) W+\left(b_{1}-k_{v}\right) V^{2}+\left(-b_{1} m_{v}+b_{1} l k_{v}-k\right) V+b_{1}(l k-m)\right] \\
V=\begin{array}{r}
Z+\frac{1}{6} b_{1}{ }^{2} m_{w}+\frac{1}{18} b_{1} k_{v} n_{v} \\
\quad+\frac{1}{18} b_{1} k_{v} m_{w}+\frac{1}{3} m_{v}-\frac{1}{9} n_{v} m_{w}+\frac{1}{36} n_{v}^{2}-\frac{1}{6} k_{v} l+\frac{1}{6} b_{1} l+\frac{1}{9} b_{1}^{2} k_{v}^{2}+\frac{1}{9} m_{w}^{2}
\end{array} \\
\left.\begin{array}{r}
W= \\
+\frac{1}{6}\left[2 b_{1} k_{v}-n_{v}+2 m_{w}\right]
\end{array}\right]\left[\frac{1}{6} b_{1}{ }^{2} m_{w}+\frac{1}{18} b_{1} k_{v} n_{v}+\frac{1}{18} b_{1} k_{v} m_{w}+\frac{1}{3} m_{v}\right. \\
\left.\quad-\frac{1}{9} n_{v} m_{w}+\frac{1}{36} n_{v}^{2}-\frac{1}{6} k_{v} l+\frac{1}{6} b_{1} l+\frac{1}{9} b_{1}^{2} k_{v}^{2}+\frac{1}{9} m_{w}^{2}\right]
\end{array}
$$

In terms of the new variables, $X, Y$ and $Z$, the curve becomes

$$
X^{2}=Y^{3}+f(Z) Y+g(Z)
$$

where

$$
\begin{aligned}
& f(Z)=Z^{3}+\alpha_{1}\left(b_{i}\right) Z+\alpha_{0}\left(b_{i}\right) \\
& g(Z)=\beta_{4}\left(b_{i}\right) Z^{4}+\beta_{3}\left(b_{i}\right) Z^{3}+\beta_{2}\left(b_{i}\right) Z^{2}+\beta_{1}\left(b_{i}\right) Z+\beta_{0}\left(b_{i}\right)
\end{aligned}
$$

The coefficients $\alpha$ and $\beta$ are expressible in terms of $\mathrm{E}_{7}$ Casimir invariants and are given in the Appendix A.

We discuss the comparison of (11) with the curve of [9] in Appendix B in more detail. Here we only present the relations ${ }^{9}$ between b's and m's:

$$
\begin{aligned}
& b_{1}=\phi \\
& b_{3}=m_{5}-m_{6}, \\
& b_{4}=m_{4}-m_{5}, \\
& b_{5}=m_{3}-m_{4}, \\
& b_{6}=m_{2}-m_{3},
\end{aligned}
$$

\footnotetext{
${ }^{9}$ It is easier to compare to [10] since they also use $\mathrm{E}_{7}$ Casimirs. It is straightforward to check that our curve is equal to that of [10] if we identify our $\mathrm{P}_{i}$ with their $\mathrm{P}_{i}$.
} 


$$
\begin{aligned}
& b_{7}=m_{1}-m_{2}, \\
& b_{8}=m_{5}+m_{6},
\end{aligned}
$$

where $\phi$ is the simple root of $\mathrm{SU}(2)$. Upon substitution in (11), we find exactly the curve of [9]. The mass parameters have a group theoretical interpretation as an orthonormal basis for the root space.

\section{Mirror Symmetry and F-theory}

In [7], it was observed that the hypermultiplet moduli space of a model constructed from the $\mathrm{E}_{6}$ extended Dynkin diagram is equal to the generalized Coulomb branch of $\mathrm{SU}(2)$ gauge theory with $\mathrm{E}_{6}$ global symmetry. In this letter, we have extended this observation to the $\mathrm{E}_{7}$ case. Given this remarkable correspondence it is natural to conjecture that there is a mirror symmetry acting on four dimensional gauge theories analogous to the mirror symmetry acting on three dimensional gauge theories [17]. However, the Coulomb branch in four dimensions is not, in general, a Hyperkähler manifold so it cannot be directly exchanged with the Higgs branch of the gauge theory which is a Hyperkähler manifold. Instead we conjecture that what is exchanged with the Higgs branch is the full four dimensional elliptically fibered space that one obtains by fibering the Seiberg-Witten torus over the usual Coulomb branch as the base. In other words, the Higgs branch gets interchanged with the four dimensional space given by the equation

$$
X^{2}=Y^{3}+f(Z) X+g(Z)
$$

where $Z$ is now interpreted as the usual coordinate on the Coulomb branch of the gauge theory. We now try to collect some evidence for this conjecture.

Firstly we can connect our generalized four dimensional mirror symmetry with the mirror symmetry that acts on three dimensional gauge theories [17] by performing a dimensional reduction of the four dimensional Seiberg-Witten theory to three dimensions. Namely, in 20] it was shown that as soon as we compactify the four dimensional photon becomes two

scalars which coordinatize the Seiberg-Witten torus thus making the full space spanned by the complex curve the natural object. It should also be noted that, in the cases where we can compare, the map between the FI parameters and the mass terms is exactly the same as in the three dimensional case [21]. Our results thus seem to be in good agreement with the results on mirror symmetry in three dimensional gauge theories.

For a more concrete connection between our mirror dual theories we now consider Ftheory compactifications [11]. The reason for this is that it is well known that the SeibergWitten theories with exceptional global symmetries can be obtained as world volume theories 
of 3-branes probing certain F-theory backgrounds [12, 13, 23, 24, 25]. Since the F-theory background looks like a set of 7-branes at strong coupling it is likely that through a sequence of T-dualities and S-dualities we can map this configuration to a brane configuration for the mirror theory. For example, starting with ordinary D3 and D7-branes, it is not difficult to imagine a sequence of dualities which would map the F-theory configuration to a configuration where a D5-brane wraps 2-cycles of an $A_{k}$ singularity. The challenge is now to extend this to the case with exceptional groups where the original configuration is strongly coupled and it is not clear what happens under duality.

We could also imagine constructing the Seiberg-Witten theory through geometrical engineering. In that case we could study the how string theory mirror symmetry acts along the lines of [16]. It is not a priori clear that the mirror theory obtained this way is the mirror theory proposed in this paper but since the three dimensional mirror symmetry can be explained in this fashion we expect a connection also in our case. If this picture is true we could take the viewpoint that what we have been doing in this paper is to "solve" the superconformal field theory with $\mathrm{E}_{7}$ global symmetry using the method of geometrical engineering and mirror symmetry as outlined in [16].

\section{Summary and Open Problems}

We have extended the observation made in [7] to $\mathrm{E}_{7}$ case: The curve corresponding to the hyperkähler quotient based on the $\mathrm{E}_{7}$ extended Dynkin diagram is equal, when it is expressed in terms of $\mathrm{E}_{7}$ Casimirs, to the generalized Coulomb branch with $\mathrm{E}_{7}$ global symmetry. The relations between FI parameters and mass parameters were obtained. The identity of the two curves led us to conjecture that mirror symmetry in the four dimensional field theories we considered should act in such a way to interchange the generalized Coulomb branch of the original theory with the Higgs branch of the dual quiver gauge theory. For evidence, we discussed the connection of the generalized Coulomb branches in four dimensions to the Coulomb branches of the three dimensional theories obtained by compactifying one dimension. We also discussed F-theory compactifications, and IIA/B mirror symmetry.

What we have shown in this article is that the complex structures of the Higgs branch and the generalized Coulomb branch are the same. To confirm the mirror hypothesis, we also need to show that the metrics are the same. It will be worth studying whether our conjecture is true in more general context. It will be also interesting to consider other quivers and study if the resulting curves can be interpreted as the generalized Coulomb branches of higher genera of some gauge theories. We hope to come back to these issues and others in [29]. 


\section{Acknowledgments}

We are very grateful to Martin Roček for many useful discussions. The work of Inyong Park

was supported in part by NSF grant PHY-97-22101 and the work of Rikard von Unge was supported in part by the Swedish Institute. 


\section{Appendix A}

The coefficients in (12) can be expressed in terms of $E_{7}$ Casimir invariants, $P_{i}$, which appear as the coefficient of $x^{56-i}$ in the expansion of $\operatorname{det}(x-v \cdot H)$. One can express $v \cdot H$ as $v \cdot H=\left(v \cdot \lambda_{1} \ldots v \cdot \lambda_{56}\right) \cdot \lambda^{\prime}$ 's are the weights of the fundamental representation. Defining ${ }^{10}$ $\chi_{n}=\frac{1}{2} \operatorname{Tr}\left[(v \cdot H)^{n}\right]$, the coefficients in (12) are

$$
\begin{aligned}
& \alpha_{1}=\frac{1}{240} \chi_{8}-\frac{11}{6480} \chi_{6} \chi_{2}+\frac{25}{2239488} \chi_{2}^{4} \\
& =-\frac{2405}{2239488} P_{2}^{4}+\frac{5}{432} P_{2} P_{6}-\frac{1}{60} P_{8} \\
& \alpha_{0}=-\frac{1}{3240} \chi_{12}+\frac{13}{81648} \chi_{10} \chi_{2}-\frac{97}{3265920} \chi_{8} \chi_{2}^{2}+\frac{19}{233280} \chi_{6}^{2} \\
& +\frac{13}{16796160} \chi_{6} \chi_{2}^{3}+\frac{103}{43535646720} \chi_{2}^{6} \\
& =\frac{63713}{60949905408} P_{2}{ }^{6}-\frac{179}{7838208} P_{2}{ }^{3} P_{6}-\frac{431}{408240} P_{2} P_{10} \\
& +\frac{19}{58320} P_{2}^{2} P_{8}-\frac{1}{5184} P_{6}{ }^{2}+\frac{1}{540} P_{12} \\
& \beta_{4}=-\frac{1}{36} \chi_{2}=\frac{1}{36} P_{2} \\
& \beta_{3}=\frac{1}{216} \chi_{6}-\frac{7}{93312} \chi_{2}^{3}=-\frac{1}{72} P_{6}+\frac{169}{93312} P_{2}^{3} \\
& \beta_{2}=-\frac{1}{2520} \chi_{10}+\frac{1}{3780} \chi_{8} \chi_{2}-\frac{13}{233280} \chi_{6} \chi_{2}^{2}+\frac{17}{67184640} \chi_{2}^{5} \\
& =\frac{1}{504} P_{10}-\frac{715}{94058496} P_{2}{ }^{5}+\frac{5}{27216} P_{2}{ }^{2} P_{6}-\frac{1}{1080} P_{2} P_{8} \\
& \beta_{1}=\frac{1}{26796} \chi_{14}-\frac{479}{18604080} \chi_{12} \chi_{2}+\frac{2857}{426202560} \chi_{10} \chi_{2}^{2} \\
& -\frac{41}{1503360} \chi_{8} \chi_{6}-\frac{6893}{13638481920} \chi_{8} \chi_{2}^{3}-\frac{9233}{70140764160} \chi_{6} \chi_{2}^{4} \\
& +\frac{1249}{121772160} \chi_{6}^{2} \chi_{2}+\frac{391207}{636317012459520} \chi_{2}^{7} \\
& =\frac{78346801}{127263402491904} P_{2}{ }^{7}-\frac{1}{3828} P_{14}-\frac{96277}{4688228160} P_{2}{ }^{2} P_{10}-\frac{1370167}{120018640896} P_{2}{ }^{4} P_{6} \\
& +\frac{56233}{5357975040} P_{2}^{3} P_{8}+\frac{1517}{29766528} P_{2} P_{6}{ }^{2}+\frac{331}{3100680} P_{2} P_{12}-\frac{91}{1378080} P_{8} P_{6} \\
& \beta_{0}=-\frac{1}{265464} \chi_{18}+\frac{18577}{21340120032} \chi_{14} \chi_{2}^{2}+\frac{397}{172020672} \chi_{12} \chi_{6} \\
& -\frac{37413577}{118529123834880} \chi_{12} \chi_{2}^{3}+\frac{551}{278737200} \chi_{10} \chi_{8}-\frac{241907}{541865116800} \chi_{10} \chi_{6} \chi_{2}
\end{aligned}
$$

\footnotetext{
${ }^{10}$ The $\chi$ 's defined here with the factor $\frac{1}{2}$ in front are more convenient because the last twenty eight weights are given by minus the first twenty eight weights as discussed below.
} 


$$
\begin{aligned}
& +\frac{62391997}{1357697236654080} \chi_{10} \chi_{2}^{4}-\frac{697}{1982131200} \chi_{8}^{2} \chi_{2}-\frac{23048029}{125712707097600} \chi_{8} \chi_{6} \chi_{2}^{2} \\
& -\frac{143590607}{108615778932326400} \chi_{8} \chi_{2}^{5}-\frac{1951}{6192744192} \chi_{6}^{3}+\frac{113390999}{1939567480934400} \chi_{6}^{2} \chi_{2}^{3} \\
& -\frac{516613213}{837893151763660800} \chi_{6} \chi_{2}^{6}+\frac{809523655}{405406222549329641472} \chi_{2}^{9} \\
= & -\frac{221}{229360896} P_{6}{ }^{3}+\frac{3794551}{397367752320} P_{2}{ }^{2} P_{8} P_{6}-\frac{71315}{7224868224} P_{2} P_{6} P_{10} \\
& +\frac{13525316017}{29663343763587072} P_{2}{ }^{6} P_{6}-\frac{55153997}{22888382533632} P_{2}{ }^{3} P_{6}{ }^{2}-\frac{473}{79639200} P_{2} P_{8}{ }^{2} \\
& +\frac{119137}{681201861120} P_{2}{ }^{3} P_{12}+\frac{45336613}{514988607006720} P_{2}^{4} P_{10}-\frac{187565459}{205995442802688} P_{2}{ }^{5} P_{8} \\
& -\frac{703}{210247488} P_{2}{ }^{2} P_{14}+\frac{73}{9556704} P_{12} P_{6}+\frac{157}{27873720} P_{10} P_{8} \\
& -\frac{142714197301}{6989762457747062784} P_{2}{ }^{9}+\frac{1}{29496} P_{18}
\end{aligned}
$$

There are the following relations between $\chi$ 's,

$$
\begin{aligned}
\chi_{4}= & \frac{\chi_{2}^{2}}{12} \\
\chi_{16}= & \frac{13}{27} \chi_{6} \chi_{10}+\frac{13}{80} \chi_{8}{ }^{2}+\frac{590}{957} \chi_{2} \chi_{14}-\frac{8567}{31320} \chi_{2} \chi_{8} \chi_{6} \\
& -\frac{15925}{103356} \chi_{2}{ }^{2} \chi_{12}+\frac{61607}{1691280} \chi_{2}{ }^{2} \chi_{6}{ }^{2}+\frac{5291}{338256} \chi_{2}{ }^{3} \chi_{10} \\
& +\frac{7397}{10824192} \chi_{2}{ }^{4} \chi_{8}-\frac{36127}{97417728} \chi_{2}{ }^{5} \chi_{6}+\frac{111449}{112225222656} \chi_{2}{ }^{8}
\end{aligned}
$$

We also have the relations between $\lambda$ 's and FI parameters. We only give the expressions for the first twenty eight weights out of fifty six since the other twenty eight $\lambda$ 's are minus the weights given below. This reflects the fact that the $\mathbf{5 6}$ is a real representation.

$$
\begin{aligned}
& v \cdot \lambda_{1}=-\frac{3}{4} b_{1}-\frac{1}{2} b_{2}-\frac{1}{4} b_{3}+\frac{1}{4} b_{5}+\frac{1}{2} b_{6}+\frac{3}{4} b_{7} \\
& v \cdot \lambda_{2}=-\frac{3}{4} b_{1}-\frac{1}{2} b_{2}-\frac{1}{4} b_{3}+\frac{1}{4} b_{5}+\frac{1}{2} b_{6}-\frac{1}{4} b_{7} \\
& v \cdot \lambda_{3}=-\frac{3}{4} b_{1}-\frac{1}{2} b_{2}-\frac{1}{4} b_{3}+\frac{1}{4} b_{5}-\frac{1}{2} b_{6}-\frac{1}{4} b_{7} \\
& v \cdot \lambda_{4}=-\frac{3}{4} b_{1}-\frac{1}{2} b_{2}-\frac{1}{4} b_{3}-\frac{3}{4} b_{5}-\frac{1}{2} b_{6}-\frac{1}{4} b_{7} \\
& v \cdot \lambda_{5}=-\frac{1}{2} b_{1}+\frac{1}{2} b_{3}+\frac{1}{2} b_{8} \\
& v \cdot \lambda_{6}=-\frac{1}{2} b_{1}-\frac{1}{2} b_{3}+\frac{1}{2} b_{8}
\end{aligned}
$$




$$
\begin{aligned}
& v \cdot \lambda_{7}=-\frac{1}{2} b_{1}+\frac{1}{2} b_{3}-\frac{1}{2} b_{8} \\
& v \cdot \lambda_{8}=-\frac{1}{2} b_{1}-b_{2}-\frac{1}{2} b_{3}+\frac{1}{2} b_{8} \\
& v \cdot \lambda_{9}=-\frac{1}{2} b_{1}-\frac{1}{2} b_{3}-\frac{1}{2} b_{8} \\
& v \cdot \lambda_{10}=-\frac{1}{2} b_{1}-b_{2}-\frac{1}{2} b_{3}-\frac{1}{2} b_{8} \\
& v \cdot \lambda_{11}=-\frac{1}{4} b_{1}+\frac{1}{2} b_{2}+\frac{1}{4} b_{3}+\frac{3}{4} b_{5}+\frac{1}{2} b_{6}+\frac{1}{4} b_{7} \\
& v \cdot \lambda_{12}=-\frac{1}{4} b_{1}-\frac{1}{2} b_{2}+\frac{1}{4} b_{3}+\frac{3}{4} b_{5}+\frac{1}{2} b_{6}+\frac{1}{4} b_{7} \\
& v \cdot \lambda_{13}=-\frac{1}{4} b_{1}+\frac{1}{2} b_{2}+\frac{1}{4} b_{3}-\frac{1}{4} b_{5}+\frac{1}{2} b_{6}+\frac{1}{4} b_{7} \\
& v \cdot \lambda_{14}=-\frac{1}{4} b_{1}-\frac{1}{2} b_{2}-\frac{3}{4} b_{3}+\frac{3}{4} b_{5}+\frac{1}{2} b_{6}+\frac{1}{4} b_{7} \\
& v \cdot \lambda_{15}=-\frac{1}{4} b_{1}-\frac{1}{2} b_{2}+\frac{1}{4} b_{3}-\frac{1}{4} b_{5}+\frac{1}{2} b_{6}+\frac{1}{4} b_{7} \\
& v \cdot \lambda_{16}=-\frac{1}{4} b_{1}+\frac{1}{2} b_{2}+\frac{1}{4} b_{3}-\frac{1}{4} b_{5}-\frac{1}{2} b_{6}+\frac{1}{4} b_{7} \\
& v \cdot \lambda_{17}=-\frac{1}{4} b_{1}-\frac{1}{2} b_{2}-\frac{3}{4} b_{3}-\frac{1}{4} b_{5}+\frac{1}{2} b_{6}+\frac{1}{4} b_{7} \\
& v \cdot \lambda_{18}=-\frac{1}{4} b_{1}-\frac{1}{2} b_{2}+\frac{1}{4} b_{3}-\frac{1}{4} b_{5}-\frac{1}{2} b_{6}+\frac{1}{4} b_{7} \\
& v \cdot \lambda_{19}=-\frac{1}{4} b_{1}+\frac{1}{2} b_{2}+\frac{1}{4} b_{3}-\frac{1}{4} b_{5}-\frac{1}{2} b_{6}-\frac{3}{4} b_{7} \\
& v \cdot \lambda_{20}=\frac{1}{2} b_{5}+b_{6}+\frac{1}{2} b_{7}+\frac{1}{2} b_{8} \\
& v \cdot \lambda_{21}=-\frac{1}{4} b_{1}-\frac{1}{2} b_{2}-\frac{3}{4} b_{3}-\frac{1}{4} b_{5}-\frac{1}{2} b_{6}+\frac{1}{4} b_{7} \\
& v \cdot \lambda_{22}=-\frac{1}{4} b_{1}-\frac{1}{2} b_{2}+\frac{1}{4} b_{3}-\frac{1}{4} b_{5}-\frac{1}{2} b_{6}-\frac{3}{4} b_{7} \\
& v \cdot \lambda_{23}=\frac{1}{2} b_{5}+\frac{1}{2} b_{7}+\frac{1}{2} b_{8} \\
& v \cdot \lambda_{24}=\frac{1}{2} b_{5}+b_{6}+\frac{1}{2} b_{7}-\frac{1}{2} b_{8} \\
& v \cdot \lambda_{25}=-\frac{1}{4} b_{1}-\frac{1}{2} b_{2}-\frac{3}{4} b_{3}-\frac{1}{4} b_{5}-\frac{1}{2} b_{6}-\frac{3}{4} b_{7} \\
& v \cdot \lambda_{26}=-\frac{1}{2} b_{5}+\frac{1}{2} b_{7}+\frac{1}{2} b_{8} \\
& v \cdot \lambda_{27}=\frac{1}{2} b_{5}-\frac{1}{2} b_{7}+\frac{1}{2} b_{8} \\
& v \cdot \lambda_{28}=\frac{1}{2} b_{5}+\frac{1}{2} b_{7}-\frac{1}{2} b_{8}
\end{aligned}
$$




\section{Appendix B}

To directly compare our curve with the one in [9], we should express our result in terms of Casimir invariants of the $\mathrm{SO}(12) \times \mathrm{SU}(2)$ subgroup of $\mathrm{E}_{7}$. More specifically, let us consider the subgroup we get by removing the simple root corresponding to $b_{2}$. Then the simple root corresponding to $b_{1}$ becomes the simple root of $S U(2)$ and the rest becomes associated with the roots of $S O(12)$. The mass parameters in [9] can be thought of as an orthonormal basis for the root space. The standard way of choosing such a basis for $S O$ algebras would in our case correspond to

$$
\begin{aligned}
& b_{3}=m_{5}-m_{6}, \\
& b_{4}=m_{4}-m_{5}, \\
& b_{5}=m_{3}-m_{4}, \\
& b_{6}=m_{2}-m_{3}, \\
& b_{7}=m_{1}-m_{2}, \\
& b_{8}=m_{5}+m_{6},
\end{aligned}
$$

and since $b_{1}$ is already orthogonal to everything else it is simply equal to the $S U(2)$ simple root

$$
b_{1}=\phi
$$

Similar relations were found in 21] for three dimensional theories. Inserting these expressions into our formulas we find that the curves are equal up to the following rescalings of the basic variables in (11)

$$
\begin{aligned}
X & \rightarrow i \frac{X}{8} \\
Y & \rightarrow-\frac{Y}{4} \\
Z & \rightarrow-\frac{Z}{2}
\end{aligned}
$$

which turns (11) into

$$
\begin{aligned}
X^{2}= & Y^{3}-\left[+2 Z^{3}+8 \alpha_{1} Z-16 \alpha_{0}\right] Y \\
& -\left[4 \beta_{4} Z^{4}-8 \beta_{3} Z^{3}+16 \beta_{2} Z^{2}-32 \beta_{1} Z+64 \beta_{0}\right],
\end{aligned}
$$

which, after a shift in $Z$ (using the notation of [9])

$$
Z \rightarrow Z+\frac{1}{6}\left(\frac{\tilde{T}_{2}^{2}}{12}+T_{4}\right)
$$

becomes exactly the curve given in [9]. 


\section{References}

[1] S. Katz, A. Klemm and C. Vafa, hep-th/9609239

[2] J. Polchinski, String Theory vol. 1,2 Cambridge

[3] M.R. Douglas and G. Moore, hep-th/9603167

[4] U. Lindström and M. Roček, Nucl. Phys. B222 (1983) 285

[5] N.J. Hitchin, A. Karlhede, U. Lindström and M. Roček, Comm. Math. Phys. 108 (1987) 535

[6] N. Seiberg, Nucl. Phys. B435 (1995) 129, hep-th/9411149

[7] U. Lindström, M. Roček and R. von Unge, hep-th/9908082

[8] J.A. Minahan and D. Nemeschansky, Nucl. Phys. B482 (1996)142

[9] J.A. Minahan and D. Nemeschansky, Nucl. Phys. B489 (1997) 24

[10] M. Noguchi, S. Terashima and S-K Yang, hep-th/9903215

[11] C. Vafa, Nucl. Phys. B469 (1996) 403, hep-th/9602022

[12] A. Sen, hep-th/9605150

[13] K. Dasgupta and S. Mukhi, hep-th/9606044

[14] T. Banks, M. Douglas and N. Seiberg, hep-th/9605199

[15] S. Kachru, A. Klemm, W. Lerche, P. Mayr and C. Vafa, Nucl. Phys. B459 (1996) 537

[16] S. Katz, P. Mayr and C. Vafa, hep-th/9706110

[17] K. Intriligator and N. Seiberg, Phys. Lett. B387 513 (1996), hep-th/9607207

[18] K. Hori, H. Ooguri and C. Vafa, hep-th/9705220

[19] P.B. Kronheimer, J. Diff. Geom. 29 (1989) 665

[20] N. Seiberg and E. Witten, hep-th/9607163

[21] J. de Boer, K. Hori, H. Ooguri, and Y. Oz, hep-th/9611063

[22] N Seiberg and E. Witten, Nucl. Phys. B431(1994) 484, hep-th/9408099 
[23] A. Johansen, hep-th/9608186

[24] M. Gaberdiel and B. Zwiebach, hep-th/9709013

[25] J. Schwarz, Phys. Lett. B360 (1995) 13, hep-th/9508143

[26] E. Witten, hep-th/9507121

[27] H. Ooguri and C. Vafa, hep-th/9511164

[28] M. Bershadsky, V. Sadov and C. Vafa, hep-th/9510225

[29] I.Y. Park and R. von Unge, work in progress 